\title{
Combining Upside and Downside Volatility in Investment Decision
}

\author{
Riccardo Bramante, Silvia Facchinetti \\ Department of Statistical Sciences, Università Cattolica del Sacro Cuore, Largo Gemelli 1, Milano, Italy \\ Email: riccardo.bramante@unicatt.it, silvia.facchinetti@unicatt.it
}

How to cite this paper: Bramante, R. and Facchinetti, S. (2022) Combining Upside and Downside Volatility in Investment Decision. Journal of Mathematical Finance, 12, 97-104. https://doi.org/10.4236/jmf.2022.121006

Received: December 22, 2021

Accepted: February 6, 2022

Published: February 9, 2022

Copyright ( 2022 by author(s) and Scientific Research Publishing Inc. This work is licensed under the Creative Commons Attribution International License (CC BY 4.0).

http://creativecommons.org/licenses/by/4.0/

\begin{abstract}
This paper deals with asset allocation decisions when the considered risk measure is directly related to the investor's level of risk aversion. It is well known that the optimal portfolio weights are considerably sensitive to how assets are ranked on the basis of their risk-return profile. We propose a procedure to construct optimal portfolios that adapt quickly to changes in risk using a time varying asset allocation model based on a modified Sharpe Ratio measure related to downside and upside risk weighted using an aversion parameter. The model is applied, as an illustrative example, to six stock markets located in Western Europe basing the analysis on monthly data covering the period January 2000-December 2020.
\end{abstract}

\section{Keywords}

Portfolio Optimization, Risk Measures, Semivariance, Asset Allocation

\section{Introduction}

Risk estimation is crucial in investment decisions [1]. In fact, the choice of different risk measures can considerably change asset risk return profile and, consequently, asset ranking in the asset allocation model. Risk measures are statistical measures that represent historical predictors of the volatility and risk of an investment, and are considered fundamental components in Modern Portfolio Theory [2].

Several risk measures have been suggested in finance to take also into consideration that risk changes through time. These measures differ in the way they treat changes in risk, in particular in terms of the historical sample period considered and of the weighting scheme applied to time series returns at the time of portfolio optimisation [3] [4]. 
Commonly used risk and performance functions in finance include: the variance, which is the earliest way of risk measurement, and allows to evaluate the volatility of an investment considering the dispersion of data from its mean; the Sharpe Ratio, which is the most widely used measure of risk-adjusted performance; Alpha, Beta and R-squared that are historical measures of past performance in comparison to a benchmark related to regression analysis. Alpha evaluates the excess return of the investment relative to the return of the benchmark index; Beta measures the systematic risk of an investment in comparison to the market as a whole; R-squared measures the percentage of a security's movement explained by movements in a benchmark index.

These measures can be used individually or together to perform a risk assessment. In selecting an appropriate risk measure, one can choose simple models, such as an equal weighting method applied to all the observations available or to a shorter but fixed sampling horizon until the point of asset allocation decision, or more complex models, such as an exponential weighted moving average or ARCH/GARCH.

Our findings contribute to the literature in the following way. We move from the classical Sharpe Ratio, that employs probabilities of negative returns, as the positive ones, weighted in the same way by the investor, to a measure which allows to overweight (underweight) risky assets on the basis of the investor decreasing (increasing) risk aversion. The model is based on a modified Sharpe Ratio that can be used to evaluate separately downside and upside risk in order to rebalance the weights of the assets in the optimal portfolio. The proposed model is applied to the relationship between the risk aversion parameter and different asset allocation strategies considering an application to real data referring to monthly returns for the period January 2000-December 2020 of six stock markets located in Western Europe: FTSE MIB (Italy), DAX (Germany), FTSE 100 (UK), CAC40 (France), IBEX (Spain) and AEX (Netherlands).

The rest of the paper is organized as follows. In Section 2 the methodology of our proposal is described, and in Section 3 an illustrative example is reported. Finally, Section 4 concludes.

\section{A Methodology for Asset Allocation}

Asset allocation is the selection of a portfolio of investments where each component is a "major" asset class rather than an individual security. Many are the optimisation techniques available that can be used in asset allocation [5]. A way to accomplish this task is to refer to the Sharpe Ratio [6] [7] introduced in Section 1 , that is defined as

$$
S_{T}=\frac{\bar{r}_{T}-r_{f}}{\sigma_{T}}
$$

where the average $\bar{r}_{T}$ and standard deviation $\sigma_{T}$ of the logarithmic returns are estimated over the sample period $\{1 ; T\}$, while $r_{f}$ is the risk free rate. The higher the Sharpe Ratio, the greater its risk-adjusted performance, and thus, the 
better the investment. The literature surrounding the Sharpe Ratio has led to many related measures such as the Information Ratio [8] and the Differential Ratio [9].

The asset allocation problem at point $T$ can be stated as

$$
\max S_{T, P}
$$

subject to

$$
\sum_{i=1}^{k} w_{i}=1 \text { and } w_{i} \geq 0
$$

where $S_{T, P}$ is the Sharpe Ratio of the portfolio $P$ and $w_{i}$ are the weights assigned to the $k$ available asset classes. Notice that the last constraint can be removed to allow for short sales, and any other constraint can be added to take into account for other asset composition restrictions.

The subset of the portfolios that will be preferred by the investor will differ according to the way risk is estimated. Moreover, in selecting a portfolio it is necessary to take into account how the investor views the trade-off between risk and return.

As for the first problem, we will focus our attention on risk thought as the failure to achieve a target or a below target outcome. In this case, we should use a statistical measure of risk which represents only downside volatility and is called semivariance

$$
\sigma_{i, T}^{2}=\frac{\sum_{t} \min ^{2}\left[0, r_{t, i}-\hat{r}_{i}\right]}{T}
$$

where $r_{t, i}$ is the logarithmic return of asset class $i$ at time $t$ and $\hat{r}_{i}$ is the target return. The use of semivariance is appealing in financial applications (see e.g. [10] [11]) and gives different statistical results, if compared for example with variance, when the distribution of the returns is asymmetric, which is the case in almost every financial returns time series (for symmetric distributions semivariance equals half of the variance). Also Markowitz said that "Analyses based on semi-variance tend to produce better portfolios than those based on variance. Variance considers extremely high and extremely low returns equally undesirable." (see [12], p.159).

In treating risk as downside volatility, we also have to consider the other part of the distribution of returns, which represents "gain" and gives a measure of upside volatility

$$
{ }_{+} \sigma_{i, T}^{2}=\frac{\sum_{t} \max ^{2}\left[0, r_{t, i}-\hat{r}_{i}\right]}{T}
$$

and so the total volatility at time $T$ of every asset class is

$$
\sigma_{i, T}^{2}={ }_{-} \sigma_{i, T}^{2}+{ }_{+} \sigma_{i, T}^{2}
$$

The problem related to this measure of risk is the selection of the target rate $\hat{r}_{i}$, which should be set exogenously and depends on the investment strategies. In this paper we assume, without loss of generality, that $\hat{r}_{i}$ equals zero for all 
the assets considered, a value commonly used which distinguishes between capital gains and losses.

In most financial applications volatility modelling must be done dynamically. Financial time series exhibit, in most cases, changes and persistence in variance and so temporal dependence in second moments must often be considered. Our approach to volatility estimation requires, in particular, to consider two conditional dynamic measures

$$
\begin{aligned}
& { }_{-} \sigma_{i, T}^{2}=E\left(r_{t, i}^{2} \mid r_{t-d, i}<\tau\right) \\
& { }_{+} \sigma_{i, T}^{2}=E\left(r_{t, i}^{2} \mid r_{t-d, i} \geq \tau\right)
\end{aligned}
$$

where $d$ is a delay parameter which can be set to one.

Having two different measures of volatility, it is necessary to define two conditional variance models. While there are many variations to model stochastic volatility, we use a relatively simple ARCH formulation of risk [13]

$$
\begin{array}{lll}
{ }_{-} \sigma_{i, T}^{2}={ }_{-} \beta_{0}+\sum_{j=1}^{P_{1}}{ }_{-} \beta_{j} \cdot \varepsilon_{t-j}^{2} & r_{t-d}<\tau \\
{ }_{+} \sigma_{i, T}^{2}={ }_{+} \beta_{0}+\sum_{j=1}^{P_{2}}{ }_{+} \beta_{j} \cdot \varepsilon_{t-j}^{2} & r_{t-d} \geq \tau
\end{array}
$$

for $i=1, \cdots, k$, and where the orders $P_{1}$ and $P_{2}$ of the ARCH processes is chosen on the basis of the minimum AIC value over a range of lag lengths. In most of the empirical analysis, values less or equal to two are sufficient to model volatility which provides enough trade off between flexibility and parsimony: for this reason the grid search is restricted to values less or equal to two.

To take into account for the investor risk tolerance, a final risk measure is defined combining downside and upside volatility by means of a weighted average, looking as follows

$$
{ }_{\lambda} \sigma_{i, T}^{2}=\lambda_{-} \sigma_{i, T}^{2}+(1-\lambda){ }_{+} \sigma_{i, T}^{2} \quad 0 \leq \lambda \leq 1
$$

where $\lambda$ is a non negative risk aversion parameter.

This approach allows to discriminate between periods in which the investor wants to overweight downside to upside volatility or vice versa.

The objective function of this new asset allocation problem can be redefined as

$$
\max _{\lambda} S_{T, P}=\frac{{ }_{P} \bar{r}_{T}-r_{f}}{{ }_{\lambda} \sigma_{P, T}}
$$

subjected to the same constraints above in (2).

\section{Empirical Analysis and Results}

The model developed in this paper is applied to the relationship between the risk aversion parameter $\lambda$ and different asset allocation strategies. For illustration purposes the data set consists of monthly returns for the period January 2000December 2020 of six stock markets chosen, as an example, within the six lead- 
ing financial centres located in Western Europe: FTSE MIB (Italy), DAX (Germany), FTSE 100 (UK), CAC40 (France), IBEX (Spain) and AEX (Netherlands). The risk free rate is the 1-year Euribor rate. For all the indices, the study of some preliminary statistics (Table 1) allows us to conclude that the series are far from being symmetric (all the reported skewness are less than -1.0) and show leptokurtosis, which is consistent with a large amount of empirical studies on the statistical properties of financial time series. The departure from normality has been confirmed for every series by the Jarque-Bera test.

In order to have an overall perspective of the data, we examine in Table 2 downside and upside volatility of the returns series: it is clear that returns more frequently fall short of breakpoint return; moreover, all the considered series experience higher downside volatility than the upside one.

The estimation of the six ARCH models was performed using numerical maximum likelihood estimation. The parameter estimates reported in Table 3, all significant at the $1 \%$ level, provide evidence concerning time varying volatility. In particular, an $\mathrm{ARCH}(1)$ model is sufficient in all of the series to describe the time dependence in the conditional volatility, both the downside and upside one.

Now, let's turn to our optimisation problem, which requires to maximise the objective Equation in (10) under the constraints defined in (2). Table 4 summarizes the results carried out using $S A S^{T M}$ for different levels of the risk aversion parameter $\lambda \in[0,1]$ at the end of 2020 using as downside and upside volatility estimates the ones retrieved from the corresponding ARCH models. Along with short-sales restrictions, an additional constraint was added to limit to a maximum level of $30 \%$ the weight assigned to each asset class.

From these results we find that changes in the risk aversion parameter modify the optimal portfolio taking into consideration the proportion of downside and upside volatility associated to each asset as stated by the ARCH models.

In Figure 1 changes in weights with respect to the "baseline" optimal portfolio when standard deviation is used are reported for the two extremes $\lambda$ parameters: ceteris paribus, optimal weights shift from stock markets and adjust accordingly to the asymmetries between downside and upside risk.

Table 1. Statistical summary of the data.

\begin{tabular}{cccccc}
\hline Index & & Standard & & Excess & Jarque \\
& Mean & Deviation & Skewness & Kurtosis & Bera \\
\hline FTSE MIB40-Italy & $-0.247 \%$ & $5.77 \%$ & -1.13138 & -0.95748 & 63.14 \\
CAC40_France & $0.103 \%$ & $4.67 \%$ & -1.61517 & 2.99319 & 202.83 \\
DAX30-Germany & $0.321 \%$ & $5.19 \%$ & -1.54234 & 2.65831 & 173.42 \\
FTSE100_UK & $0.003 \%$ & $3.84 \%$ & -1.90499 & 5.66651 & 487.62 \\
IBEX-Spain & $-0.078 \%$ & $5.11 \%$ & -1.13907 & 1.65533 & 82.93 \\
AEX-Netherlands & $-0.015 \%$ & $5.07 \%$ & -1.94169 & 5.03655 & 423.01 \\
\hline
\end{tabular}


Table 2. Downside and upside volatility of the returns series.

\begin{tabular}{cccc}
\hline Index & $\begin{array}{c}\text { Downside } \\
\text { Volatility }\end{array}$ & $\begin{array}{c}\text { Upside } \\
\text { Volatility }\end{array}$ & \% Downside \\
\hline FTSE MIB40-Italy & $4.6110 \%$ & $3.4516 \%$ & $64.09 \%$ \\
CAC40_France & $3.7540 \%$ & $2.7717 \%$ & $64.72 \%$ \\
DAX30-Germany & $4.0499 \%$ & $3.2423 \%$ & $60.94 \%$ \\
FTSE100-UK & $3.1363 \%$ & $2.2072 \%$ & $66.88 \%$ \\
IBEX-Spain & $4.0101 \%$ & $3.1451 \%$ & $61.92 \%$ \\
AEX-Netherlands & $4.1925 \%$ & $2.8291 \%$ & $68.71 \%$ \\
\hline
\end{tabular}

Table 3. ARCH volatility models.

\begin{tabular}{ccc}
\hline Index & Downside Volatility & Upside Volatility \\
& $\beta_{1}$ & $\beta_{1}$ \\
\hline FTSE MIB40-Italy & 0.210622 & 0.237809 \\
CAC40-France & 0.191541 & 0.266914 \\
DAX30-Germany & 0.196246 & 0.319617 \\
FTSE100-UK & 0.181620 & 0.226903 \\
IBEX_Spain & 0.199735 & 0.215180 \\
AEX-Netherlands & 0.197460 & 0.276253 \\
\hline
\end{tabular}

Table 4. Optimal portfolios.

\begin{tabular}{ccccccc}
\hline & \multicolumn{5}{c}{ Weights } \\
\cline { 2 - 7 }$\lambda$ & FTSE MIB40 & CAC40 & DAX30 & FTSE100 & bIBEX & AEX \\
\hline 0.10 & Italy & France & Germany & UK & Spain & Netherlands \\
0.20 & $6.11 \%$ & $20.20 \%$ & $20.17 \%$ & $26.54 \%$ & $11.60 \%$ & $15.39 \%$ \\
0.30 & $6.16 \%$ & $20.22 \%$ & $20.65 \%$ & $26.19 \%$ & $11.81 \%$ & $14.99 \%$ \\
0.40 & $6.18 \%$ & $20.23 \%$ & $21.45 \%$ & $25.62 \%$ & $12.15 \%$ & $14.37 \%$ \\
0.50 & $6.19 \%$ & $20.23 \%$ & $21.79 \%$ & $25.37 \%$ & $12.29 \%$ & $14.12 \%$ \\
0.60 & $6.21 \%$ & $20.22 \%$ & $22.10 \%$ & $25.16 \%$ & $12.42 \%$ & $13.90 \%$ \\
0.70 & $6.22 \%$ & $20.21 \%$ & $22.37 \%$ & $24.96 \%$ & $12.53 \%$ & $13.71 \%$ \\
0.80 & $6.22 \%$ & $20.19 \%$ & $22.62 \%$ & $24.78 \%$ & $12.63 \%$ & $13.54 \%$ \\
0.90 & $6.23 \%$ & $20.18 \%$ & $22.85 \%$ & $24.62 \%$ & $12.72 \%$ & $13.39 \%$ \\
\hline
\end{tabular}

In this way, the model can support the investor in rebalancing the weights assigned to the assets modifying the risk aversion parameter. Having a look, as an example, to German DAX market the risk aversion parameter reduces the weight when low values of $\lambda$ are used whereas optimal weight allocation results in an increase when $\lambda$ moves to its upper bound level. 




Figure 1. Optimal Portfolios weights comparison.

\section{Concluding Remarks}

In this paper we developed an asset allocation model based on a modified Sharpe Ratio measure. Classical Sharpe Ratio implies that the probability of negative returns, as the probability of positive returns, is weighted in the same way by the investor. In order to evaluate separately downside and upside risk we consider a measure which permits to overweight (underweight) risky assets on the basis of the investor decreasing (increasing) risk aversion. In this way, the model can be used to rebalance the weights of the assets in the optimal portfolio.

Generalizations could be taken into consideration in the future to expand the results presented in this paper. Extending our approach to other forms of riskadjusted measures certainly represents an obvious area of development of the present contribution. A further possible extension goes in the direction of using nonlinear averaging methods to assess portfolio volatility starting from the estimates of the positive/negative parts.

\section{Conflicts of Interest}

The authors declare no conflicts of interest regarding the publication of this paper.

\section{References}

[1] Chen, S.-N. and Brown, S.J. (1983) Estimation Risk and Simple Rules for Optimal Portfolio Selection. Journal of Finance, 38, 1087-1093. https://doi.org/10.1111/j.1540-6261.1983.tb02284.x

[2] Markowitz, H. (1952) Portfolio Selection. Journal of Finance, 7, 77-91. https://doi.org/10.1111/j.1540-6261.1952.tb01525.x

[3] Cvitanic, J. and Karatzas, I. (1999) On Dynamic Measures of Risk. Finance and Stochastics, 3, 451-482. https://doi.org/10.1007/s007800050071

[4] Giovannini, A. and Jorion, P. (1989) The Time Variation of Risk and Return in the 
Foreign Exchange and Stock Markets. Journal of Finance, 44, 307-325. https://doi.org/10.1111/j.1540-6261.1989.tb05059.x

[5] Michaud, R.O. (2008) Efficient Asset Management: A Practical Guide to Stock Portfolio Optimization and Asset Allocation. Oxford University Press, New York.

[6] Sharpe, W.F. (1964) Capital Asset Prices: A Theory of Market Equilibrium under Conditions of Risk. Journal of Finance, 19, 425-442. https://doi.org/10.1111/j.1540-6261.1964.tb02865.x

[7] Sharpe, W.F. (1994) The Sharpe Ratio. Journal of Portfolio Management, 21, 49-58. https://doi.org/10.3905/jpm.1994.409501

[8] Chincarini, L.B. and Daehwan, K. (2008) Another Look at the Information Ratio. Journal of Asset Management, 8, 284-295. https://doi.org/10.1057/palgrave.jam.2250085

[9] Moody, J., Wu, L., Liao, Y. and Saffell, M. (1998) Performance Functions and Reinforcement Learning for Trading Systems and Portfolios. Journal of Forecasting, 17, 441-470.

[10] Lewis, A.L. (1990) Semivariance and the Performance of Portfolios with Options. Financial Analysts Journal, 46, 67-76. https://doi.org/10.2469/faj.v46.n4.67

[11] Estrada, J. (2008) Mean-Semivariance Optimization: A Heuristic Approach. Journal of Applied Finance, 18, 57-72. https://doi.org/10.2139/ssrn.1028206

[12] Markowitz, H.M. (1952) Portfolio Selection. John Wiley \& Sons, New York. https://doi.org/10.2307/2975974

[13] Xekalaki, E. and Degiannakis, S. (2010) ARCH Models for Financial Applications. John Wiley \& Sons, New York. https://doi.org/10.1002/9780470688014 